\title{
Modelling the interplay between the shear layer and leading edge suction during dynamic stall
}

\author{
Julien Deparday ${ }^{1}$ and Karen Mulleners ${ }^{1, \text { a) }}$ \\ UNFoLD, Institute of Mechanical Engineering, École Polytechnique Fédérale de \\ Lausanne (EPFL), CH-1015 Lausanne, Switzerland
}

(Dated: 29 August 2019)

The dynamic stall development on a pitching airfoil at $R e=10^{6}$ was investigated by time-resolved surface pressure and velocity field measurements. Two stages were identified in the dynamic stall development based on the shear layer evolution. In the first stage, the flow detaches from the trailing edge and the separation point moves gradually upstream. The second stage is characterised by the roll up of the shear layer into a large scale dynamic stall vortex. The two-stage dynamic stall development was independently confirmed by global velocity field and local surface pressure measurements around the leading edge. The leading edge pressure signals were combined into a single leading edge suction parameter. We developed an improved model of the leading edge suction parameter based on thin airfoil theory that links the evolution of the leading edge suction and the shear layer growth during stall development. The shear layer development leads to a change in the effective camber and the effective angle of attack. By taking into account this twofold influence, the model accurately predicts the value and timing of the maximum leading edge suction on a pitching airfoil. The evolution of the experimentally obtained leading edge suction was further analysed for various sinusoidal motions revealing an increase of the critical value of the leading edge suction parameter with increasing pitch unsteadiness. The characteristic dynamic stall delay decreases with increasing unsteadiness and the dynamic stall onset is best assessed by critical values of the circulation and the shear layer height which are motion independent.

a)Electronic mail: karen.mulleners@epfl.ch 
Modelling the interplay between the shear layer and leading edge suction

\section{INTRODUCTION}

The sudden stall from a dynamically pitching airfoil is a classic example of unsteady flow separation with applications in rotary aircraft, rapidly manoeuvring fixed wing aircraft, and wind turbines. The unsteady change in angle of attack leads to a delay in stall onset and an increase in the maximum attainable lift with respect to the static response. Although stall delay and lift overshoot sound beneficial, dynamic stall is generally a phenomenon that is to be avoided. Large excursions of the highly unsteady aerodynamic loads following stall onset decrease the aerodynamic efficiency and yield strong vibrations and increasing structural forces and bending moments that cause fatigue and potential damage to the airfoil and the structure supporting it $^{1-3}$.

Early research on dynamic stall was focussed on understanding the main evolution and succession of flow phenomena and the effect of the kinematic parameter on the aerodynamic forces and pitching moment of the airfoil ${ }^{4-8}$. Smoke flow visualisation was used to qualitatively describe the coherent vortex structures developing during dynamic stall. The development of unsteady pressure sensors made it possible to obtain accurate spatially and temporally resolved surface pressure distributions and measure high frequency turbulent flow oscillations ${ }^{9-13}$. The surface pressure measurements revealed the presence and continued growth of a suction peak at the leading edge during dynamic stall development. The use of time-resolved particle image velocimetry allowed for accurate flow field measurements that were analysed to reveal the dominant role of the dynamic stall vortex and its characteristic time scales ${ }^{14-18}$. Recent high-fidelity large eddy simulations ${ }^{19,20}$ as well as direct numerical simulations ${ }^{21}$ of dynamic stall on a pitching airfoil show excellent agreement with experimental results ${ }^{22}$. Numerical simulations provide the complete flow field information, including velocity field data, airfoil surface pressure and surface friction data, but they remain computationally expensive for Reynolds numbers above $5 \times 10^{5}$.

To protect the integrity of the airfoil and the supporting structure and avoid loss of aerodynamic performance, it is essential to accurately predict the dynamic stall development and onset. The response of the flow to unsteady changes in angle of attack is non-linear and depends on many parameters such as the airfoil profile, inflow Reynolds number, motion kinematics. If the angle of attack is increased fast enough to an angle above the static stall limit, flow will separate from the leading edge $\mathrm{e}^{23-29}$. It thus seems natural to search for the 
indication of massive dynamic flow separation near the leading edge. As early as 1959, Evans $\&$ Mort $^{30}$ presented evidence of a correlation between the minimum value of the leading edge surface pressure coefficient and the steepness of the adverse pressure gradient at stall. Leishman \& Beddoes ${ }^{31}$ defined their stall onset criterion for incompressible flows based on the work of Evans \& Mort ${ }^{30}$. They related the critical leading edge pressure to a critical normal force coefficient to derive a practical stall onset criterion for their dynamic stall model that predicts the aerodynamic loads for unsteady angle of attack variations. The influence of the unsteady conditions that were taken into account are twofold. Due to the unsteady change in angle of attack, there is a lag in the increase of the normal force coefficient with angle of attack and there is a lag in the leading edge pressure response with respect to the normal force coefficient. As a consequence, the critical leading edge pressure will be reached at a higher value of the normal force coefficient, which in turn will be reached at a higher angle of attack with increasing pitch rate. Both effects are included in a deficiency function that describes the first order lag of the normal force coefficient with an empirical time constant that depends solely on the Mach number. The influence of progressive trailing edge separation, which is present in many dynamic stall cases even if they are dominated by leading edge separation, is represented in the Beddoes-Leishman model as a variation of the slope of the normal force coefficient curve in function of the angle of attack. The correction is based on Kirchhoff's law and includes an empirical evolution of the trailing edge separation location with angle of $\operatorname{attack}^{32}$.

Noteworthy improvements of the Beddoes-Leishman model include the works of Niven \& Galbraith $^{33}$ and Sheng et al. ${ }^{34}$. Niven \& Galbraith ${ }^{33}$ introduced an additional empirical time constant adding to the lag of the normal force coefficient representing the finite time required for the disturbed flow to develop into a vortex. They also presented an extension of the Kirchhoff law in the unsteady regime to improve the predictions of the dynamic stall model for scenarios where trailing edge flow reversal is non negligible. According to Niven \& Galbraith ${ }^{33}$, a more aesthetic prediction of dynamic stall onset should be based on a quantifiable feature of the unsteady trailing edge flow reversal behaviour. Sheng et al. ${ }^{34}$ criticise the uncertainties associated with the upper and lower bounds of the critical leading edge pressure from the Evans \& Mort correlation ${ }^{30}$ and proposes a new criterion for stall onset that is based on a critical angle of attack instead of a critical normal force coefficient. This new criterion relies on a linear variation of the dynamic stall onset angle of attack with 


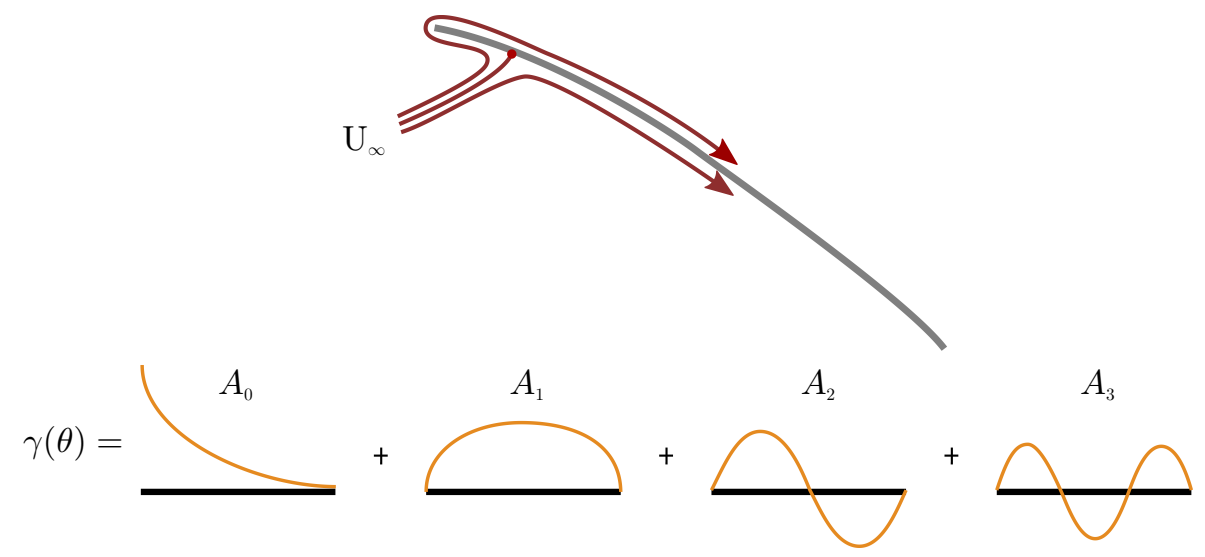

FIG. 1. Schematic representation of the flow around the leading edge of a thin airfoil and the associated vortex strength distribution $\gamma$ of the vortex sheet representing the airfoil's camber line broken into the first 4 terms of the Fourier series. (Inspired by Katz \& Plotkin ${ }^{39}$ ).

pitch-rate for ramp-up motions which does not hold for lower pitch rates and in scenarios where unsteady trailing edge flow reversal is observed.

A more recent stall onset criterion that has rapidly gained popularity is the critical leading edge suction parameter criterion proposed by Ramesh \& al. ${ }^{35}$. This criterion was initially developed for application in discrete vortex models ${ }^{36,37}$. It is based on the idea that an airfoil can support a maximum amount of leading edge suction. If this maximum limit is exceeded, vorticity is released from the leading edge, accumulates, and forms a leading edge or dynamic stall vortex. The critical value of the leading edge suction parameter depends on the airfoil shape and Reynolds number and is independent of the motion kinematics for scenarios where no trailing-edge separation is present ${ }^{38}$.

The leading edge suction parameter proposed by Ramesh \& al. ${ }^{35}$ is related to the first term of the Fourier series of the distribution of the vortex sheet strength along the camber line in thin airfoil theory. Thin airfoil theory determines the aerodynamic force on an airfoil as the result of the potential pressure field induced by a vortex sheet with strength distribution $\gamma(\theta, t)$ along the airfoil's camber line ${ }^{39}$. The vortex sheet strength distribution represents the deviation of the flow due to the presence of the airfoil and it is calculated such that there is no net normal induced velocity along the camber line. The circulation distribution 
for a generalised camber line can be described as a Fourier series:

$$
\gamma(\theta, t)=2 U_{\infty}\left(A_{0}(t) \frac{1+\cos \theta}{\sin \theta}+\sum_{n=1}^{\infty} A_{n}(t) \sin n \theta\right)
$$

where the angle $\theta$ is related to the chord-wise coordinate $x$ along the camber line through:

$$
x=\frac{c}{2}(1-\cos \theta) .
$$

The time-dependent Fourier coefficients $A_{n}(t)$ are determined to satisfy the Kutta condition at the trailing edge (figure 1). The first Fourier coefficient $A_{0}$ represents the leading edge suction peak resulting from the acceleration of the flow around the airfoil's leading edge ${ }^{40}$. The critical values of the dynamic stall onset parameters summarised above are generally well defined for dynamic stall scenarios where trailing edge flow reversal is absent or suppressed. However, in most occurrences of dynamic stall, progressive trailing edge separation and flow reversal play a significant role $12,19,20,41$. In this paper, we consider experimental data of deep dynamic stall on a pitching airfoil where stall onset is the result of the roll up of a free shear layer at the interface between the outer flow and a region of flow reversal near the airfoil's suction surface. We will analyse the interplay between the unsteady development of the free shear layer and the leading edge suction during dynamic stall development based on experimental time-resolved velocity field and surface pressure data. Based on the results, we will propose a theoretical model of the leading edge suction parameter with the temporal evolution of the shear layer height as the quantifiable feature of the unsteady trailing edge flow reversal. The characteristic time-scales of the evolution of the shear layer and the leading edge suction will be identified and compared and critical values with regard to dynamic stall onset will be identified.

\section{EXPERIMENTAL SETUP}

The experimental data used for the current analysis stems from experiments that were conducted in a closed-circuit, low-speed wind tunnel of the German Aerospace Centre (DLR) in Göttingen. The data from this measurement campaign was previously used to study the onset and development of dynamic stall ${ }^{14,43,44}$. The wind tunnel had an open test section with a rectangular nozzle of $0.75 \mathrm{~m} \times 1.05 \mathrm{~m}$. Simultaneous unsteady surface pressure and timeresolved velocity field measurements using particle image velocimetry (PIV) were conducted 


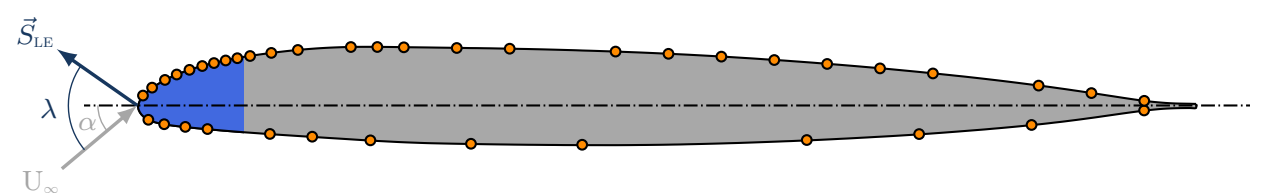

FIG. 2. Positions of pressure sensors on the airfoil shown by orange dots. The blue shaded region defines the leading edge section that is considered to calculate the leading edge suction vector $S_{\mathrm{LE}}$ and its chord-wise component $F_{\mathrm{S}}$ that is used to calculate the experimental leading edge suction parameter. (Reprinted from Deparday \& Mulleners ${ }^{42}$ with the permission of AIP Publishing.)

for a sinusoidally pitching airfoil. The airfoil had a OA209 cross section profile, a chord length of $c=0.3 \mathrm{~m}$, and an aspect ratio of 5 . The airfoil was pitching around its quarter chord axis. The OA209 airfoil profile is a typical helicopter blade profile and it represents a class of airfoils that are potentially affected by dynamic stall in reality. It was placed in a uniform flow of $U_{\infty}=50 \mathrm{~m} / \mathrm{s}\left(R e_{c}=9.2 \times 10^{5}\right)$. Measurements were taken in the cross-sectional plane at the model mid-span.

The unsteady surface pressure distribution was determined using 41 surface-mounted pressure transducers, which were sampled at a rate of $6 \mathrm{kHz}$ and integrated to obtain the lift history. The pressure data from the 13 pressure taps in the first $10 \%$ of the chord was integrated to obtain the leading edge suction $S_{\mathrm{LE}}$ vector (figure 2).

The velocity field was measured using stereo PIV with an acquisition rate of $1500 \mathrm{~Hz}$. The PIV system consisted of a diode-pumped Nd:YAG laser (Lee Laser, LDP-200MQG Dual) that emitted laser pulses with an energy of approximately $12 \mathrm{~mJ}$ per pulse and two high speed CMOS cameras (Photron Ultima APX-RS) with 1024 px $\times 1024$ px sensors. The vertical plane at model mid-span was illuminated by the laser from above and the cameras were mounted downstream of the airfoil in a stereoscopic set-up alongside the wind tunnels diffuser. The width of the field of view covered the entire chord for the relevant angle of attack range. Due to experimental limitations, valid velocity information was only available down to approximately $3 \mathrm{~mm}$ or $0.01 \mathrm{c}$ above the airfoils surface. The time-resolved PIV data were evaluated using a multi-grid algorithm with a final interrogation window size of $32 \mathrm{px} \times 32 \mathrm{px}$ and an overlap of approximately $80 \%$ yielding a spatial resolution of $1.2 \mathrm{~mm}$, or 0.004c. The detection probability of a valid displacement in the PIV data was higher than $90 \%$. The remaining spurious vectors were detected and replaced using the automated 
Modelling the interplay between the shear layer and leading edge suction

post-processing algorithm of Garcia ${ }^{45}$. The relative measurement error was estimated to be less than 0.02 every where. More details about the PIV error estimation can be found in Mulleners \& Raffel ${ }^{43}$. The velocity fields were rotated into the airfoil frame of reference prior to analysis, with the $x$-axis along chord, the $y$-axis along span and the $z$-axis upward perpendicular to the chord.

The angle of attack of the airfoil was varied sinusoidally around a mean incidence $\alpha_{0}$, close to the static stall angle, with an amplitude $\alpha_{1}$, and an oscillation frequency $f_{\text {osc }}$. A large range of dynamic stall cycles was obtained by varying these three motion parameters. The reduced frequencies $k=\pi f_{\text {osc }} c / U_{\infty}$ of all cases are between 0.05 and 0.1 . The instantaneous effective unsteadiness $\dot{\alpha}_{\mathrm{ss}}^{\star}$ was introduced previously by Mulleners \& Raffel ${ }^{14}$ as a single representative parameter to describe the influence of the motion parameters describing a sinusoidal pitching motion on dynamic stall development. It is defined as the rate of change of the angle of attack at the moment when the static stall angle is exceeded and it is non-dimensionalised by the convective time: $\dot{\alpha}_{\mathrm{ss}}^{\star}=\dot{\alpha}_{\mathrm{ss}} c / U_{0}$.

\section{DYNAMIC STALL LIFE CYCLE}

The typical dynamic stall flow development within a single pitching cycle is represented in figure 3 by means of selected instantaneous velocity fields. The colour map represents the out-of-plane vorticity component $\omega_{y}=\partial u / \partial z-\partial w / \partial x$. The angle of attack in this example varies sinusoidally around a mean angle of attack of $\alpha_{0}=20^{\circ}$ with an amplitude of $\alpha_{1}=8^{\circ}$ and a reduced pitching frequency $k=0.05$. We consider the start of the pitching cycle when the angle of attack is lowest. The first half of the cycle is referred to as the pitch-up motion and the second half as the pitch-down motion. The maximum angle of attack in the presented case is $\alpha_{\max }=28^{\circ}$ and well beyond the static stall angle of attack $\alpha_{\mathrm{ss}}=21.4^{\circ}$ under the given circumstances. The temporal history of the lift coefficient during the dynamic stall cycle is presented in figure 4 and displays the characteristic features of deep dynamic stall including stall delay to an angle of attack beyond the static stall angle, lift overshoot with respect to the maximum static lift, and large load fluctuations following dynamic stall. The timing of the individual snapshots in figure 3 is indicated in the lift history. The evolution of the circulation during the selected pitching cycle is also included in figure 4 . The solid line represents the positive circulation calculated by integrating all positive vorticity in the field 


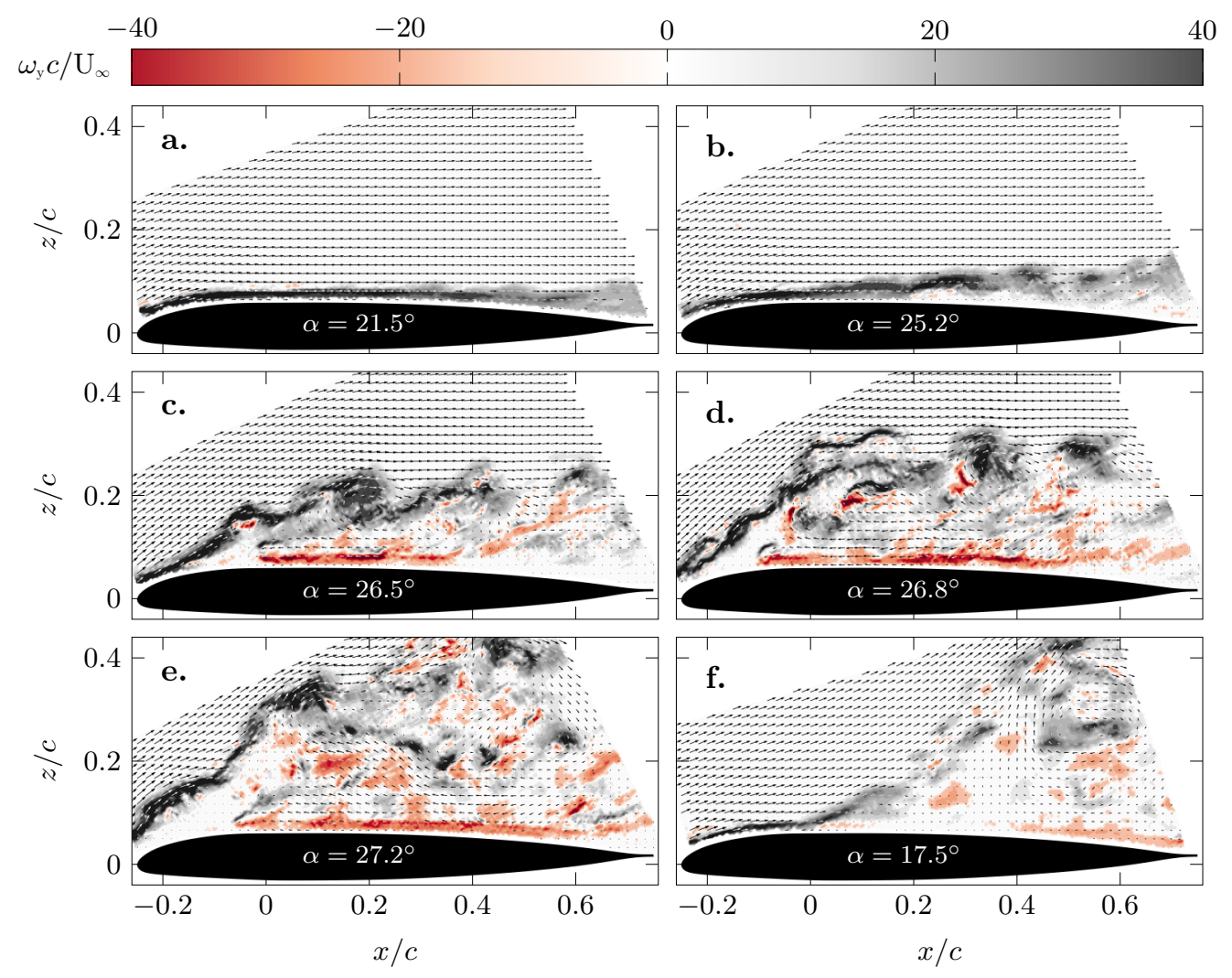

FIG. 3. Selected instantaneous velocity and vorticity field snapshots during a single sinusoidal pitching cycle described by $\alpha_{0}=20^{\circ}, \alpha_{1}=8^{\circ}, k=0.05$, and normalised effective unsteadiness $\dot{\alpha}_{\mathrm{ss}}^{\star}=0.013$. The snapshots were taken at $t / T=0.28$ (a.), 0.36 (b.), 0.40 (c.), 0.41 (d.), 0.43 (e.), 0.80 (f.) within the same pitching cycle. The timing of the snapshots is also indicated in the lift history in figure 4. (Modified from Deparday \& Mulleners ${ }^{42}$ with the permission of AIP Publishing.)

of view and the dashed line represents the negative circulation calculated by integrating all negative vorticity in the field of view. The leading edge vortex has positive signed circulation and the trailing edge vortex negative circulation. The evolution of the shear layer is characterised in terms of its dimensionless average shear layer height $\Delta z^{\star}$ (figure 4c.). It is defined as the dimensionless chord-wise averaged chord-normal distance between the shear layer location and the airfoil's suction surface at any given time ${ }^{43}$. The shear layer location is identified in individual velocity field snapshots by the location of clock-wise rotating shear layer vortices that in turn are identified using Eulerian vortex criteria ${ }^{14,46}$.

The temporal evolution of the dynamic stall development has been discussed in detail in previous publications ${ }^{14,43,44}$ and is summarised here for reference. From the beginning of 


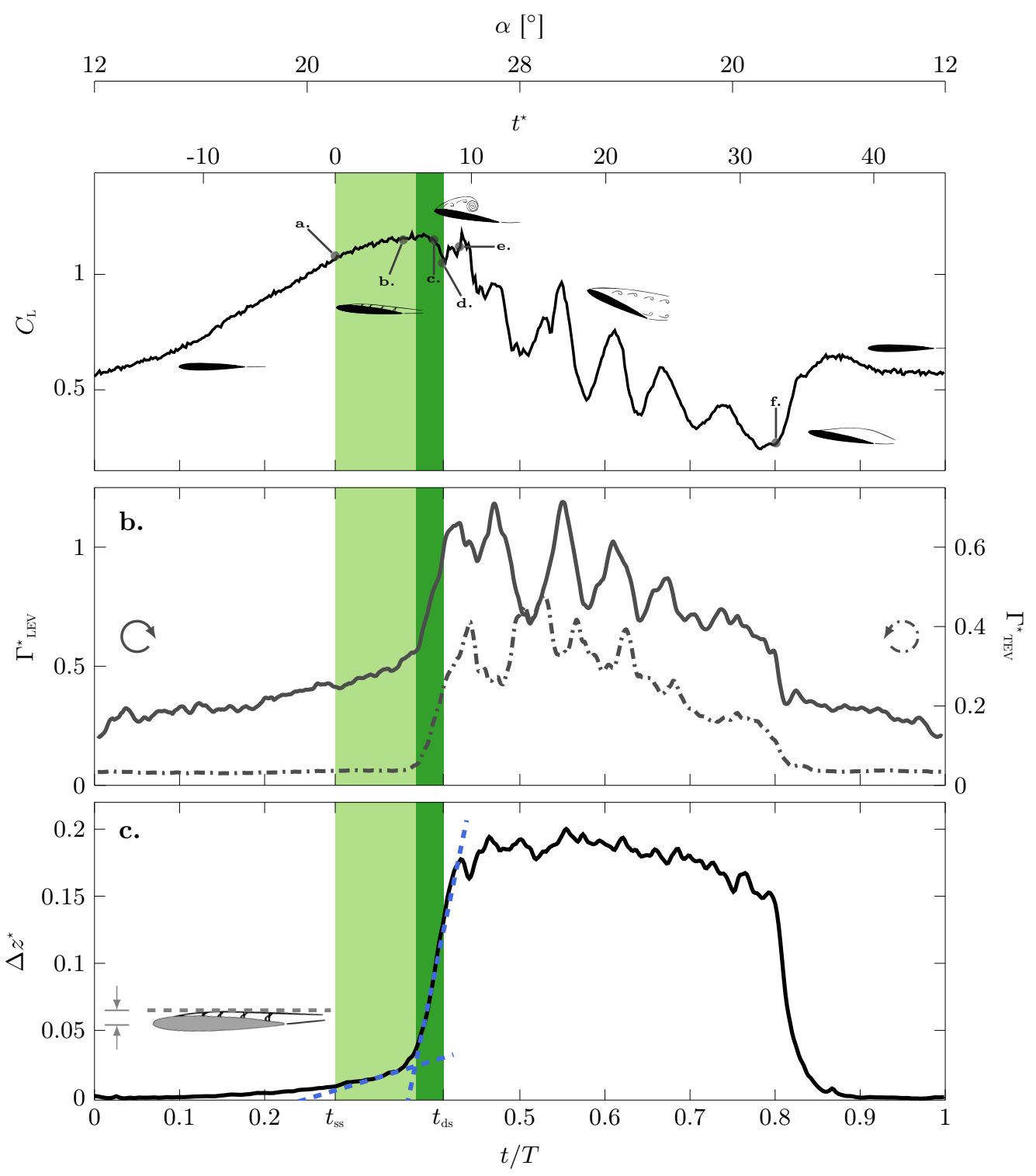

FIG. 4. Temporal evolution of the lift coefficient (a.), positive and negative circulation (b.), and chord-wise average chord-normal shear layer distance (c.) during an individual pitching cycle described by $\alpha_{0}=20^{\circ}, \alpha_{1}=8^{\circ}, k=0.05$, and $\dot{\alpha}_{\mathrm{ss}}^{\star}=0.013$. The labels a. to f. in the lift history indicate the timing of the velocity and vorticity field snapshots in figure 3 . The shaded regions refer to the stall development stages between the time when the static stall angle of attack is exceeded $t_{\mathrm{ss}}$ and the dynamic stall onset is reached $t_{\mathrm{ds}}$. The dashed lines in c. represent the linear fits of $\Delta z^{\star}$ characteristic for the primary instability and the vortex formation stage. 
the pitch-up motion until the static stall angle is reached at $t_{\mathrm{ss}}$, the flow is attached to the airfoil (figure 3a.). During this attached flow stage, the spatially averaged shear layer height from instantaneous velocity fields is close to zero and the lift coefficient and positive circulation increase with increasing angle of attack. Beyond the static stall angle $\alpha_{\mathrm{ss}}$, the lift keeps increasing and the flow starts to detach at the trailing edge (figure 3b.). The trailing edge separation point moves upstream with increasing angle of attack (figure 3b.-c.). At the interface between the region of flow reversal near the trailing edge and the free stream, a shear layer forms where positive vorticity is concentrated. The vorticity contained in the shear layer is accumulated in small-scale co-rotating vortices, as a result of a primary shear layer instability or Kelvin-Helmholtz instability ${ }^{21,47}$. The reversed flow region expands with increasing angle of attack pushing the shear layer further away from the airfoil's suction surface. During this initial spread of the trailing edge separation region, the average shear layer height increases approximately linearly in time and the lift coefficient increases at a lower rate than below the static stall angle (figure 4). Later in the cycle, the shear layer vortices start to pair, which initiates the roll-up of the shear layer into a large scale coherent dynamic stall vortex (figure 3c.-d. $)^{44}$. The period between the time at which the static stall angle is exceeded up to the time at which the shear layer starts to roll up is called the primary instability stage and is highlighted in the figures that present time histories by the light colour shading. The period during which the shear layer rolls up into a primary stall vortex is called the vortex formation stage and is highlighted in the figures by the dark colour shading.

At the beginning of the shear layer roll-up, the lift coefficient reaches a maximum. During the shear layer roll-up, the lift coefficient decreases while the positive circulation keeps increasing. The average shear layer height now increases at a rate 15 times higher than during the primary instability stage. Another characteristic feature of the second stage of stall development is the emergence of negative vorticity at the airfoil surface underneath the shear layer (figure 3c.). The induced velocity due to the growing positive circulation of the primary stall vortex pushes the negative vorticity towards the leading edge and ultimately leads to the self-induced detachment of the dynamic stall vortex (figure $3 \mathrm{~d}$.) ${ }^{14,48}$. The timing of the pinch-off of the dynamic stall vortex is referred to as the dynamic stall onset $\left(t_{\mathrm{ds}}\right)$. After pinch-off, the dynamic stall vortex is convected downstream and the airfoil is fully stalled (figure 3e.). During the fully stalled stage of the dynamic stall life cycle, the average shear 
layer height is maximum and strong fluctuations in the lift coefficient and the circulation are observed which are attributed to the formation and shedding of successive stall vortices. Near the end of the pitch-down motion, for an angle of attack lower than the static stall angle $\alpha_{\mathrm{ss}}$, the flow reattaches to the airfoil starting from the leading edge (figure 3f.). During flow reattachment, the lift coefficient increases and the shear layer height and circulation values decrease. At the end of the pitch-down motion, the flow is fully reattached and the lift coefficient has relaxed to the same value obtained at the beginning of the sinusoidal motion.

The time-resolved velocity field data reveals that dynamic stall development is a two-stage process. A first stage, named the primary instability stage, is characterised by an increasing region of flow reversal indicated by a linear growth of the chord normal distance between the shear layer and the airfoil's suction surface. Flow reversal occurs in the boundary layer directly at the airfoil's surface and is not immediately followed by flow separation in a dynamic stall life cycle ${ }^{33,49}$. During this first stage, the lift overshoot with respect to the static stall limit is generated and there is limited interaction between the individual vortices in the shear layer. A second stall development stage, named the vortex formation stage, is characterised by the roll-up of the shear layer into a large coherent dynamic stall vortex. During this second stage, the flow actually separates and the free shear layer no longer follows the airfoil contour. The transition between the primary instability stage and the vortex formation stage was determined directly from the velocity field data as the intersection between the linear fitting curves of the temporal evolution of the average height of the shear layer (figure 4c.). The dynamic stall onset was determined by the first peak in the time development coefficient of the proper orthogonal decomposition mode that represents the dynamic stall vortex. The timing of this peak coincides with the departure of the Lagrangian saddle point, which marks the intersection between the attracting and repelling material lines that bound the dynamic stall vortex, away from the surface ${ }^{14}$. The emergence and trajectories of Lagrangian saddle points are not only reliable indicators of the pinch-off or lift-off of coherent leading edge or wake vortices but they also leave a distinct footprint in the surface pressure distribution and the force history ${ }^{50-53}$.

Time-resolved velocity field data allows for accurate determination of dynamic stall onset but it cannot be readily utilised as an observable parameter to serve as prediction input for practical applications such as flow control or gust alleviation ${ }^{54-58}$. Inspired by the idea 
of Niven \& Galbraith ${ }^{33}$ that an aesthetic prediction of dynamic stall onset and the critical leading edge suction should be based on a quantifiable feature of the unsteady trailing edge flow reversal, we aim to derive a theoretical model of the leading edge suction parameter based on the height of the shear layer. Prior to proposing a theoretical model of the leading edge suction parameter based on thin airfoil theory and the evolution of the shear layer height, we will first describe the interplay between the shear layer development and the evolution of the experimentally determined leading edge suction parameter.

\section{LEADING EDGE SUCTION DURING DYNAMIC STALL}

\section{A. Leading edge suction parameter}

The leading edge suction parameter for our experimental data is derived from the leading edge suction vector $\vec{S}_{\mathrm{LE}}$ which is determined by integrating the pressure signals from the 13 unsteady pressure sensors located in the front $10 \%$ of the airfoil (figure 2$)^{42}$. Approximately $40 \%$ of the lift and $90 \%$ of the chord-wise force component during the pitch-up motion are generated by the front $10 \%$ of the airfoil. Figure 5 presents the phase-averaged evolution of the leading edge suction vector during pitch-up for the example dynamic stall case presented in figure 3. The phase-averaged quantities were obtained by averaging over more than 35 pitching cycles. As a measure for the variability of the signals between cycles, we have added the minimum and maximum envelopes. Prior to the dynamic stall vortex pinch-off, the repeatability of the pressure measurements and leading edge suction vector is high and the phase-averaged evolution is representative for all pitching cycles. During the stalled stage, cycle-to-cycle variations of the leading edge suction vector rapidly increase, mainly due to variations in the vortex shedding process and timing.

The leading edge suction vector is characterised by a magnitude $S_{\mathrm{LE}}$ (figure 5a.) and an angle $\lambda$ relative to the inflow velocity direction (figure 5b.). The experimental leading edge suction parameter $A_{0}$ (figure $5 \mathrm{c}$.), is calculated based on the chord-wise projection of the leading edge suction vector according to Katz \& Plotkin ${ }^{39}$ :

$$
A_{0, \exp }=\sqrt{\frac{2}{\pi}\left|S_{L E}\right| \cos (\lambda-\alpha)} .
$$

The magnitude of the leading edge suction vector $S_{\mathrm{LE}}$ increases during the attached flow and the primary instability stage and decreases during the vortex formation stage. The 
leading edge suction magnitude reaches a maximum exactly at the transition between the primary instability and the vortex formation stage and a local minimum at the dynamic stall onset. The timing of the stages is determined directly from the velocity field data that does not include information from within the boundary layer and the leading suction vector only includes information from the front $10 \%$ of the airfoil. The concurrence between crucial changes in the flow field and the leading edge suction is remarkable, it confirms the existence of a two-stage stall development, and it is encouraging to further explore the potential of stall criteria based the leading edge suction.

The orientation of the leading edge suction vector gives us additional information about the location of the region that contributes most to the leading edge suction. During the attached flow and primary instability stage, $\lambda \approx 85^{\circ}$ and the leading edge suction vector is approximately perpendicular to the incoming flow (figure 5b.). This indicates that the topology of the flow around the leading edge has not yet been altered during the primary instability stage despite the growing region of flow reversal near the trailing edge. As a consequence, if the pitching direction is reversed prior to the onset of the vortex formation stage, massive flow separation and load hysteresis can be significantly reduced. This condition has been identified in literature as light dynamic stall ${ }^{7,14}$. During the vortex formation stage, the suction angle slightly decreases first, i.e. suction shifts towards the leading edge, and then increases to $\approx 90^{\circ}$ at dynamic stall onset. At the beginning of the fully stalled stage, the primary dynamic stall vortex is convected downstream and the leading edge suction vector will roughly point in the direction of the vortex indicated by the rapid increase of the leading edge suction angle up to $\approx 120^{\circ}$ when the maximum angle of attack is reached.

The leading edge suction vector $\vec{S}_{\mathrm{LE}}$ gives an idea about the spatiotemporal pressure evolution around the leading edge during the different stages of the dynamic stall life cycle and is further condensed in a single leading edge suction parameter that is proposed as a simple but robust parameter that governs leading edge or dynamic stall vortex formation by Ramesh \& al. ${ }^{35}$. The evolution of the leading edge suction parameter is similar to the evolution of the magnitude of the leading edge suction vector with an increase during the attached phase and the primary instability stage, a maximum at the transition between the primary instability and the vortex formation stages, and a decrease during the vortex formation stage (figure 5c.). The main difference between the evolution of the leading edge suction magnitude and parameter is the absence of a secondary local suction peak due to 

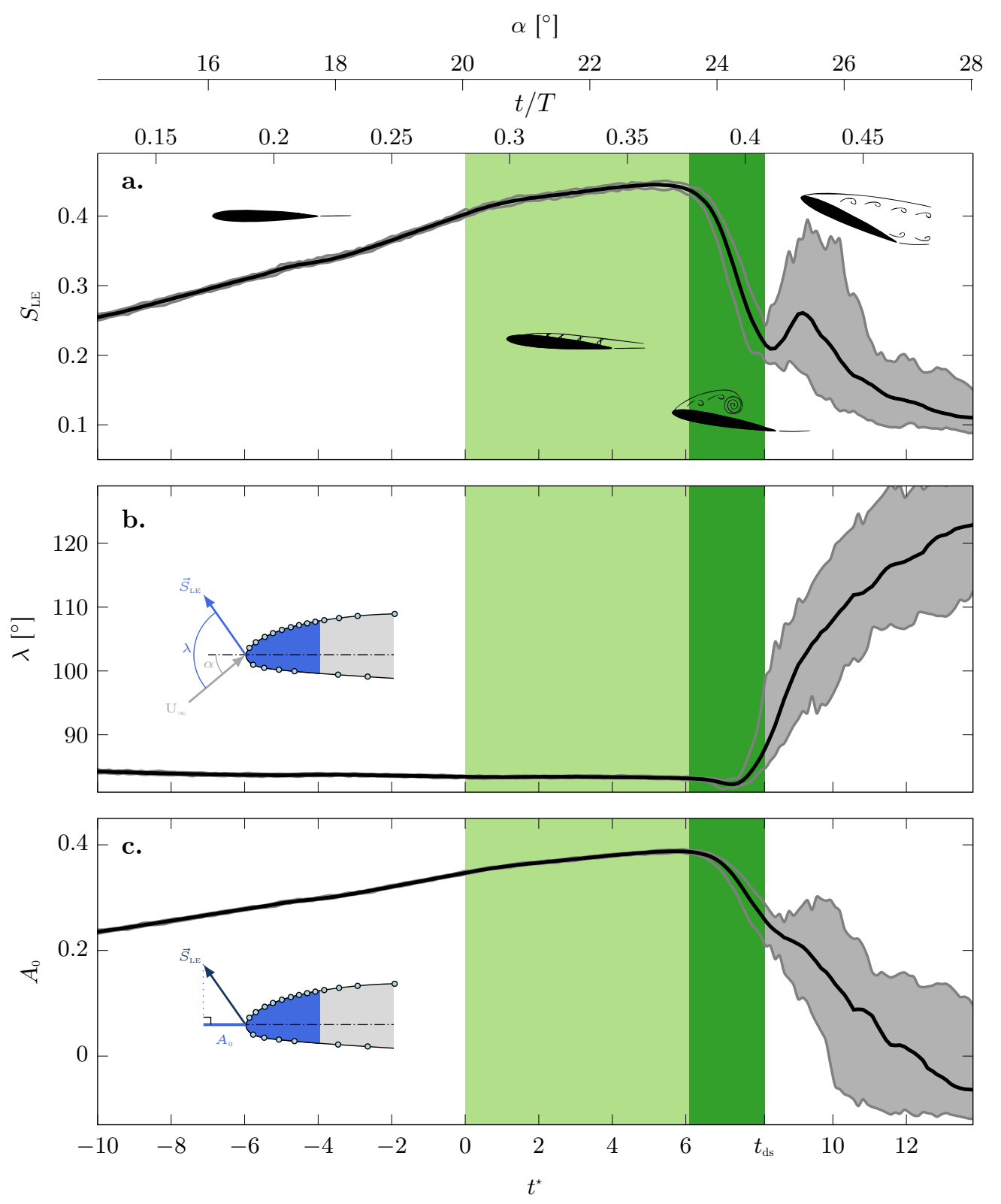

FIG. 5. Magnitude $S_{\mathrm{LE}}$ of the leading edge suction force (a.), its orientation $\lambda$ relative to the inflow velocity (b.), and the leading edge suction parameter (c.) for a pitching motion described by $\alpha_{0}=20^{\circ}, \alpha_{1}=8^{\circ}$, and $k=0.05$. The thick black line represents the phase average and the grey region is delineated by the minimum and maximum envelopes.

the shedding of the primary stall vortex in the suction parameter because the downstream convection of the vortex does not seem to affect the chord-wise component of the leading edge suction. 


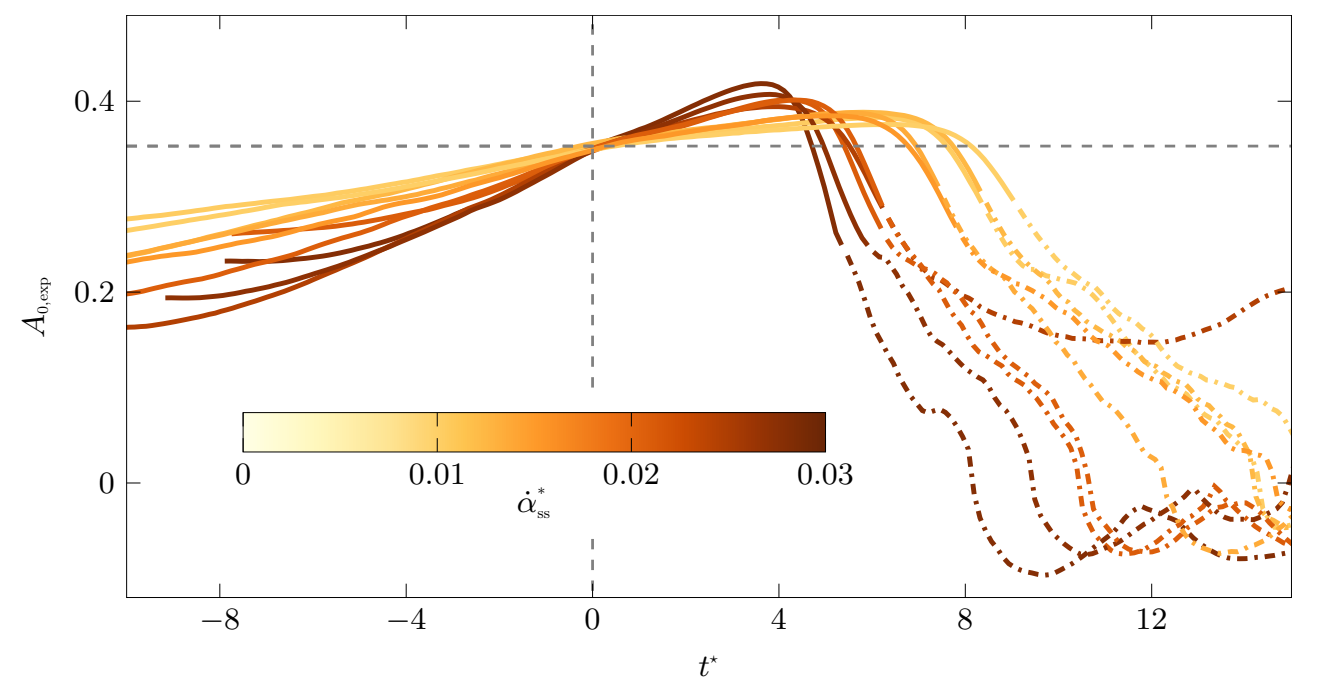

FIG. 6. Temporal evolution of the measured phase-averaged leading edge suction parameter $A_{0}$ during the dynamic stall development stage for different normalised effective unsteadiness $\dot{\alpha}_{\mathrm{ss}}^{\star}$. The static stall angle is reached at $t^{\star}=0$. Full lines represent the evolution up to dynamic stall onset and dashed lines represent the post-stall evolution.

\section{B. Critical values and characteristic time scales}

The leading edge suction parameter represents the suction maintained at the leading edge. There is a critical amount of suction that the leading edge can sustain and above which the airfoil stalls ${ }^{59}$. According to Ramesh $\&$ al. ${ }^{38}$, the critical value of the leading edge suction parameter depends mainly on the airfoil shape and the Reynolds number and is independent of the motion kinematics except when high degrees of trailing-edge flow separation occur during the motion. The ideal dynamic stall prediction parameter has a critical value at dynamic stall onset that is largely independent of the flow and motion conditions and can easily be determined or derived for various airfoil geometries. The leading edge suction parameter has strong potential in this direction, but we need to further explore how the temporal evolution of the leading edge suction parameter and its critical values vary with the unsteadiness of the motion kinematics for dynamic stall cases where stall onset is the result of the roll up of the free shear layer bounding the region of flow reversal near the airfoil surface.

The phase-averaged temporal evolution of the experimental leading edge suction parameter for various sinusoidal pitching motions with different amplitudes, mean angles of attack, and 


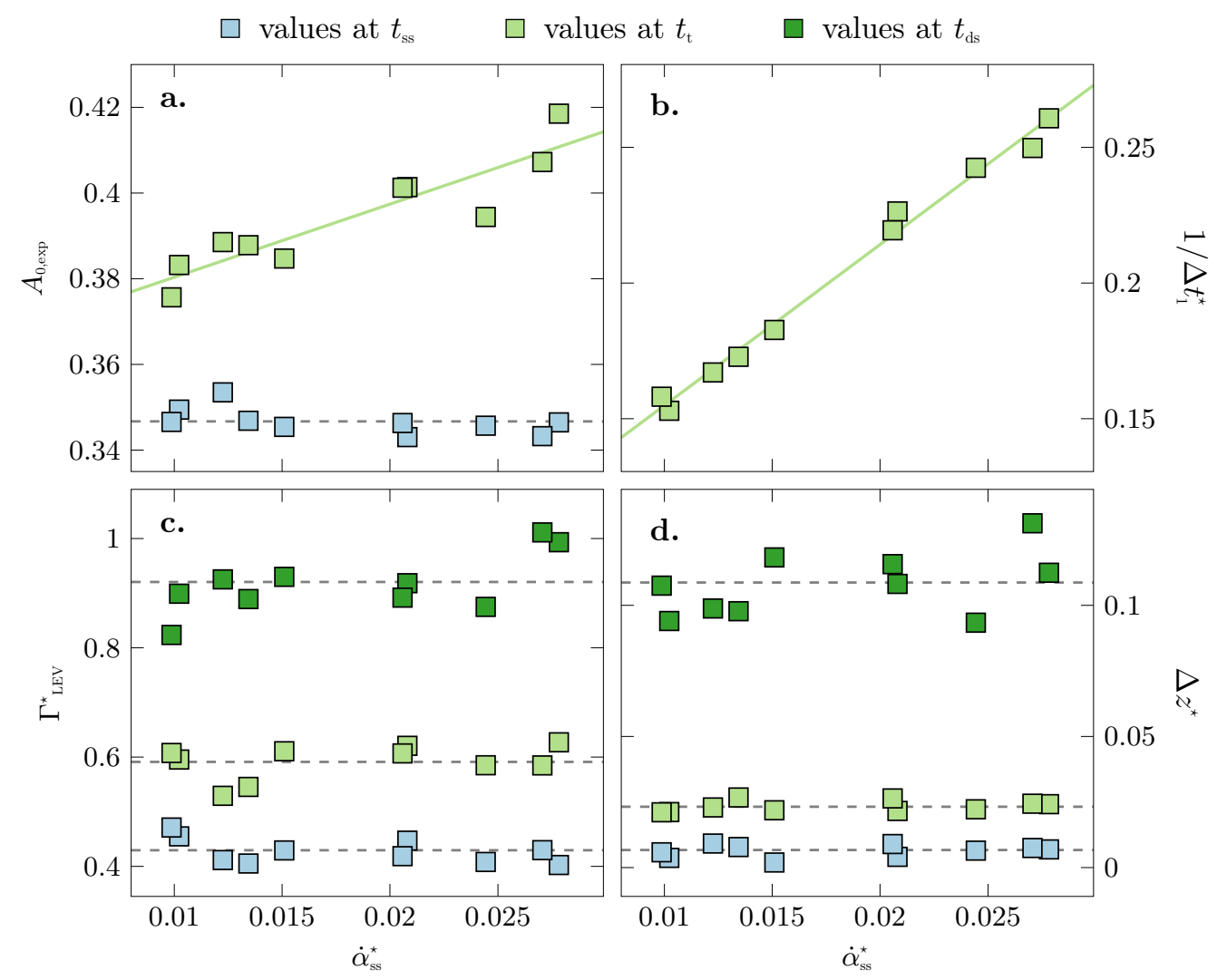

FIG. 7. Characteristic values of the leading edge suction parameter (a.), the inverse of the stall delay (b.), the positive circulation associated with the leading edge or dynamic stall vortex (c.), and the chord-wise averaged chord-normal shear layer height (d.) in function of the normalised effective unsteadiness. The colour of the symbols indicate whether the values represent the situation at the start of the dynamic stall development $t_{\mathrm{ss}}$, at the transition between the two stall development stages $t_{\mathrm{t}}$, or at dynamic stall onset $t_{\mathrm{ds}}$.

pitching frequencies are presented in figure 6 . The line colour indicates the unsteadiness of the harmonic pitching motions in terms of the normalised effective unsteadiness. The latter is defined as the dimensionless pitch rate when the static stall angle of attack is exceeded. It was found to be a suitable scaling parameter for the timing of stall onset by Mulleners $\&$ Raffel $^{14}$ and Mller-Vahl et al. ${ }^{28}$. All curves are aligned in time with respect to the time when the static stall angle is exceeded which is considered the start of the dynamic stall development stage. The lines are solid up to the occurrence of dynamic stall and dashed thereafter.

The general evolution of the leading edge suction parameter is similar for the different 
motions. It starts with a linear increase during the primary instability stage followed by a linear decrease during the vortex formation stage. For a non-separated flow, the value of the leading edge suction parameter is related to the angle of attack and all curves intersect at $t^{\star}=0$, where the angle of attack equals the static stall angle of attack. During the primary instability stage, the slope of the leading edge suction parameter increases with increasing normalised effective unsteadiness due to the increasing rate of change of the angle of attack around $t^{\star}=0$. The maximum leading edge suction increases and is reached earlier for higher pitch rates. During the vortex formation stage, which starts sooner for higher normalised effective unsteadiness, the slope increases with increasing unsteadiness. The maximum or critical value of the leading edge suction parameter, which is reached for all motions at the transition between the two stall development stages, is not constant but increases with increasing unsteadiness. This in line with the recent observations by SureshBabu et al. ${ }^{60}$. The characteristic values of the leading edge suction parameter at the start of the stall development stage and the maximum values at the start of the vortex formation stage are summarised in figure $7 \mathrm{a}$ in function of the normalised effective unsteadiness. The maximum value of the leading edge suction parameter increases approximately linearly with increasing unsteadiness and the same holds for the reciprocal of the convective time at which the maximum value is reached (figure $7 \mathrm{~b}$ ). The higher the local pitch rate, the faster a critical leading edge suction value is reached. At the same time, an increasing lag in the onset of the instability that leads to the roll-up of the shear layer seems to increase the value of the critical amount of suction that is obtained at the leading edge. The deficiency function introduced in Leishman \& Beddoes' dynamic stall onset criterion takes into account that a critical value of the leading edge pressure will be reached at a higher value of the normal force coefficient which in turn will be reached at a higher angle of attack with increasing pitch rate, but it does not consider an increase of the value of the critical leading edge pressure.

The time at which the leading edge suction parameter reaches a maximum coincides with the transition between the two dynamic stall development stages as determined previously based on the evolution of the shear layer height. The durations of the two stall development stages in terms of convective time units are independently quantified based on the global velocity field data and based on the surface pressure date at the leading edge and results are compared in figure 8 . We obtain the same resulting time delays for the primary instability stage $\Delta t_{1}{ }^{\star}$ 


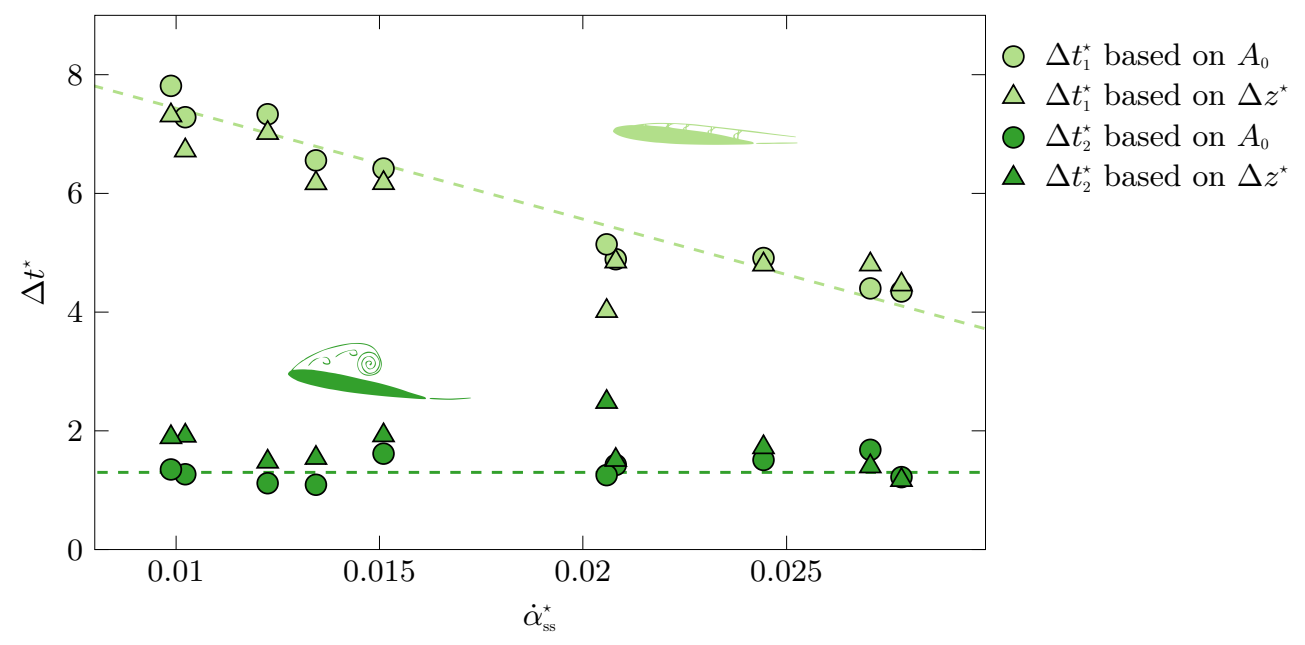

FIG. 8. Time duration of the two stall development stages for different normalised effective unsteadiness measured independently by local pressure measurements $\left(A_{0}\right)$ and by global velocity field measurement $\left(\Delta z^{\star}\right)$.

and the vortex formation stage $\Delta t_{2}{ }^{\star}$ for both measurements. This result is not trivial. The velocity field measurements do not include information from within the boundary layer near the airfoil's surface. The leading edge suction parameter was calculated taking into account only the surface pressure measurements in the front $10 \%$ of the airfoil profile. The fact that we are able to identify two stall development stages in these two independently measured quantities and extract the same associated characteristic time scales for the stall stages clearly demonstrates the direct connection between the evolution of the shear layer height $\Delta z^{\star}$ and the leading edge suction parameter $A_{0}$. This results also underlines the potential of the latter to serve as an observable parameter for the prediction of dynamic stall onset and control applications.

The remaining drawback of the leading edge suction parameter as a universal dynamic stall onset indicator, is the variation of its critical value with the unsteadiness of the pitching motion. By linking the leading edge suction to other characteristic parameters that obtain limiting critical values at dynamic stall onset or at the transition between the two stall development stages, we aim to contribute to the applicability and generalisability of a dynamic stall criterion based on the leading edge suction parameter and to a better understanding why dynamic stall happens when it happens.

The durations of the stall development stages reach characteristic values that aid to under- 
Modelling the interplay between the shear layer and leading edge suction

stand the influence of the motion kinematics on the stall delay. The duration of the vortex formation stage is independent of the normalised effective unsteadiness (figure 8). This stage is characterised by the roll-up of the shear layer into a coherent dynamic stall vortex and is predominantly driven by viscous interactions which dependent on the Reynolds number but not on the motion kinematics. This constant time delay corresponds to the empirical time constant that was introduced by Niven \& Galbraith ${ }^{33}$ as an extension of the Beddoes-Leishman model.

The governing mechanisms during the primary instability stage are different and yield a decreasing time delay with increasing increasing unsteadiness even though the critical leading edge suction increases (figure 8). We can also present the reciprocal of the time delay, which decreases linearly with increasing effective unsteadiness (figure $7 \mathrm{~b}$ ).

Even though the dependence of the stall delay with the motion kinematics is clear, it does not provide a general critical value above which stall onset is triggered. Motion independent critical values are observed for the shear layer height and positive circulation associated with leading edge vortices (figure $7 \mathrm{c}-\mathrm{d}$ ). For both parameters, the values at the start of dynamic stall development $\left(t_{\mathrm{ss}}\right)$, at the transition between the two stall development stages $\left(t_{t}\right)$, and at dynamic stall onset $\left(t_{\mathrm{ds}}\right)$ are constant for all different motions. The values at these key points in the dynamic stall life cycle increase with increasing convective time and progression in the stall development. At dynamic stall onset, the standard deviation of the values from different motions is larger and some minor dependence on the unsteadiness could still be present for the circulation at $t_{\mathrm{ds}}$. As previously concluded, the flow development during the vortex formation stage is governed by viscous interactions and the convective time duration of this stage is independent of the motion kinematics. The relevant critical values for dynamic stall onset are the threshold values at $t_{\mathrm{t}}$ as they trigger the roll-up of the shear layer into the dynamic stall vortex. An increase in circulation within the free shear layer will increase the mutual interaction between small scale shear layer vortices ${ }^{13}$ and the tendency of the shear layer to roll-up. The shear layer can only roll up if its chord normal distance to the airfoil surface is large enough. This explains why both parameters reach critical values at the start of the shear layer roll-up independent of the motion kinematics. The higher the effective unsteadiness of the pitching motion, the sooner the critical circulation threshold is reached and the shorter the stall delay associated with the primary stall development stage. Critical thresholds of the circulation and the shear layer height are reliable indicators of stall 
onset for the airfoil profile and Reynolds number considered here. The threshold values are independent of the motion kinematics but the parameters are not easily accessible outside of the laboratory environment. The leading edge suction parameter is a robust and more practical parameter that could even be determined in flight and serve as an observable for in-situ flow control applications where early detection of the inception of dynamic stall is essential. Its applicability and reliability hinges upon an a priori knowledge of the critical value of the leading edge suction in function of the pitching motion parameters. A loworder model based on thin airfoil theory is proposed in the follow section to predict the critical values and the evolution of the leading edge suction parameter during dynamic stall development by including the effect of trailing edge flow reversal.

\section{THEORETICAL MODEL OF THE LEADING EDGE SUCTION PARAMETER}

\section{A. Comparison with thin airfoil theory}

The leading edge suction parameter is the first coefficient of the Fourier series of the circulation distribution along the chord. It is calculated by integrating the local induced velocity $v_{\text {ind }}(x, t)$ normal to the surface:

$$
A_{0, \text { theor }}=-\frac{1}{\pi} \int_{0}^{\pi} \frac{v_{\text {ind }}(x, t)}{U_{\infty}} d \theta \text {. }
$$

The induced normal velocity represents the deviation of the parallel free stream due to the presence of the airfoil. For a pitching airfoil, the normal induced velocity has three contributions:

$$
v_{\text {ind }}(x, t)=-U_{\infty} \sin \alpha-\dot{\alpha} x_{\mathrm{p}}+\frac{\partial \eta}{\partial x} U_{\infty} \cos \alpha
$$

The first term represents the effect of the angle of attack, the second term represents the influence of the unsteady pitching motion, where $x_{\mathrm{p}}$ is the location of the airfoil's pivot point, and the last term represents the influence of the camber of the airfoil, where $\eta(x)$ is the chord-normal coordinate of the camber line along the chord.

For a sinusoidal pitching motion around the quarter chord, the theoretical leading edge 


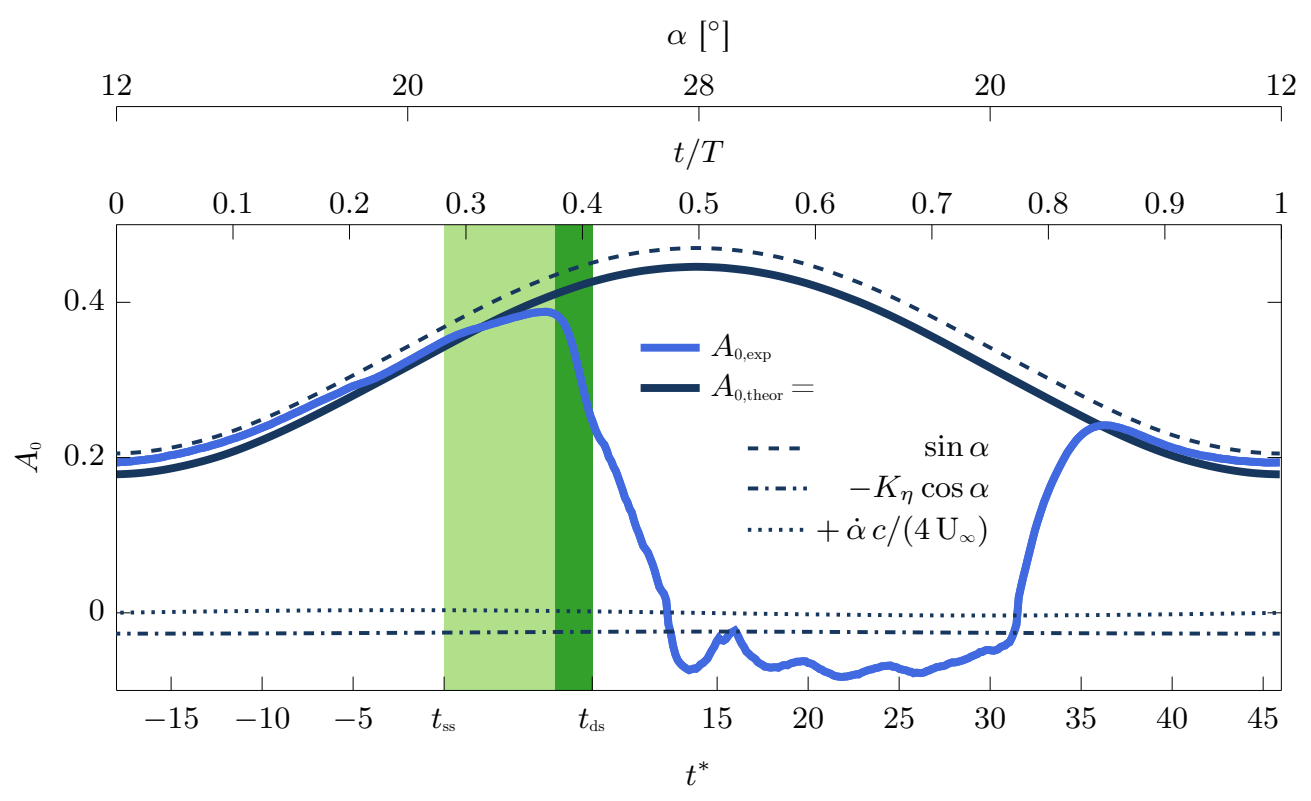

FIG. 9. Comparison of the experimental $\left(A_{0, \exp }\right)$ and theoretical $\left(A_{0, \text { theor }}\right)$ leading edge suction parameter for the same motion as presented in figure 3. The influence of the different terms in equation (6) are presented by the dashed lines.

suction parameter reads:

$$
A_{0, \text { theor }}=\sin \alpha+\dot{\alpha} \frac{c}{4 U}-K_{\eta} \cos \alpha
$$

where

$$
K_{\eta}=\frac{1}{\pi} \int_{0}^{\pi} \frac{\partial \eta(\theta)}{\partial x} d \theta
$$

represents the influence of the airfoil's camber.

The influence of the angle of attack, pitching rate, and camber terms during a pitching motion are presented in figure 9 with dashed lines. The theoretical leading edge suction parameter is presented by the dark line and the experimental phase-averaged leading suction parameter by the bright line. The time on the abscissa is non-dimensionalised by the convective time. The time over a pitching period and the angle of attack are indicated on the top. The two stages of the dynamic stall development are marked by the shaded areas. The first term $\sin \alpha$ is an order of magnitude higher than the other terms. It ranges from 0.2 to 0.4 while the second term composed of the pitch rate is almost zero and the third term varies around -0.02 . The first term is the dominant term in the model of the theoretical leading edge suction parameter and evolves sinusoidally with a maximum for the 


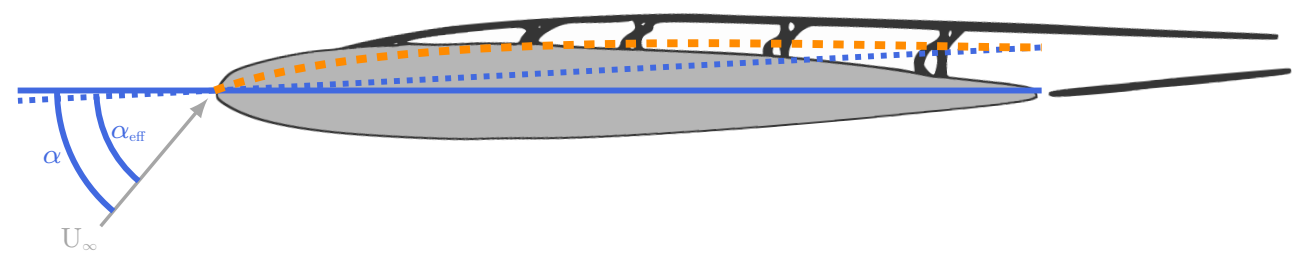

FIG. 10. Schematic representation of the influence of the shear layer growth on the effective angle of attack $\left(\alpha_{\mathrm{eff}}\right)$ and the effective camber.

maximum angle of attack. The experimental leading edge suction parameter and its model are in close agreement during the attached flow stage and the reattachment stage.

During the first stage of the dynamic stall development, the experimental leading edge suction parameter $A_{0, \exp }$ increases at a slower rate than during the attached flow stage. This is not correctly represented by the theoretical leading edge suction parameter that increases at a higher rate than the experimental leading edge suction parameter. The values of both at the time the static stall angle of attack is reached $\left(t^{\star}=0\right)$ are almost identical. Due to different growth rate during the primary instability stage, the model overestimates the leading edge suction during stall development. At the transition between the primary instability and the vortex formation stage, the experimental leading edge suction parameter reaches a maximum which is not represented by the potential model. During the vortex formation stage, the theoretical leading edge suction parameter continues to increase while the experimental leading edge suction drops to values close to zero when the flow is separated.

When the flow reversal region spreads from the trailing to the leading edge during the primary instability stage, the curvature of the flow is effectively decreased reducing the acceleration of the flow around the leading edge and the leading edge suction. This effect is not included in the model presented by equation (6). Thin airfoil theory predicts well the increase of the experimental leading edge suction parameter when the flow is attached but needs to be extended to include the growth of the flow reversal region during the primary instability stage. 


\section{B. Influence of shear layer on the leading edge suction parameter}

The experimental results revealed that the shear layer plays an important role in the dynamic stall development. We propose an improvement of the theoretical model of the leading leading edge suction parameter by taking into account a twofold influence of the evolution of the shear layer, being a change in the effective camber and a change of the effective angle of attack of the airfoil (figure 10).

The position of the shear layer above the airfoil's suction surface virtually increases the thickness of the airfoil. The height of the shear layer is added to the position of the airfoil's suction surface relative to the camber of the airfoil. During the primary instability stage, the shear layer height which is defined here as the vertical distance between the shear layer and the airfoil's suction surface, is larger near the trailing edge than at the leading edge where the height is almost zero (figure $3 \mathrm{~b}$ ).

If the height of the shear layer is taken into account, the effective thickness $t_{\text {eff }}$ is the sum of the airfoil's geometrical thickness $t_{\text {geo }}$ and the shear layer height $t_{\mathrm{sl}}$ :

$$
\forall x, t_{\mathrm{eff}}(x)=t_{\mathrm{geo}}(x)+t_{\mathrm{sl}}(x)
$$

Using the definition of the purely geometric camber for the airfoil $\eta_{\text {geo }}$

$$
z_{\text {upper,geo }}=\eta_{\text {geo }}+\frac{1}{2} t_{\text {geo }}
$$

and the definition of the effective camber for the virtual airfoil profile $\eta_{\text {eff }}$

$$
z_{\text {upper, eff }}=\eta_{\text {eff }}+\frac{1}{2} t_{\text {eff }}
$$

we calculate the effective camber of the virtual airfoil profile as a function of the shear layer height and geometric camber:

$$
\eta_{\mathrm{eff}}=\eta_{\mathrm{geo}}+\frac{1}{2} t_{\mathrm{sl}}
$$

We now modify the third term of the potential model of the leading edge suction parameter (equation (6)) by using the virtually altered airfoil profile according to:

$$
\begin{aligned}
K_{\eta_{\mathrm{eff}}} & =K_{\eta}+\frac{1}{2 \pi} \int_{0}^{\pi} \frac{\partial t_{\mathrm{sl}}(\theta)}{\partial x} d \theta \\
& =K_{\eta}+\Delta z^{\star}
\end{aligned}
$$


where $\Delta z^{\star}$ is the experimentally determined dimensionless chord-wise average of the shear layer height.

The first term of this equation (12) represents the influence of the geometric camber according to equation (7) and the second term represents the influence of the shear layer height on the effective camber. The growth of the shear layer leads to an increase in effective camber. The chord-wise variation of the shear layer height is approximated here by a linear increase from zero at the leading edge to $2 \Delta z^{\star}$ at the trailing edge. The linear increase of the shear layer height is a first order approximation that seems reasonable during the primary instability stage (figure $3 \mathrm{~d}$ ).

The additional consequence of the development of the shear layer is a change in the effective angle of attack. The shear layer has a maximum height at the trailing edge which leads to a change in camber but also an upward shift of the virtual trailing edge and a decrease of the effective angle of attack which is schematically represented in figure 10. The effective angle of attack is given by:

$$
\begin{aligned}
\alpha_{\text {eff }} & =\alpha-\alpha_{\mathrm{sl}} \\
& =\alpha-\tan \Delta z^{\star} .
\end{aligned}
$$

This calculation of the effective angle of attack could be further extended to include the effect of varying pivot point locations based on the recent work by Li et al. ${ }^{61}$.

The modified model for the leading edge suction parameter taking into account the influence of the shear layer growth during stall development is given by:

$$
A_{0, \text { theor }}=\sin \alpha_{\text {eff }}+\dot{\alpha} \frac{c}{4 U}-K_{\eta} \cos \alpha_{\text {eff }}-\Delta z^{\star} \cos \alpha_{\text {eff }} .
$$

By replacing the geometric angle of attack in equation (6) by the effective angle of attack and adding a new term, the change of camber due to the presence of the shear layer is taken into account. The first term in equation (15) is still an order of magnitude higher than the other terms and is presented in figure 11 by the dashed line, along with the newly added term $\Delta z^{\star} \cos \alpha_{\text {eff }}$ in the dot-dashed line. Figure 11 also presents the final result of the modified leading edge suction parameter model $A_{0, \text { theor }}$ by the dark solid line and the experimentally obtained leading edge suction parameter $A_{0, \exp }$ by the bright solid line.

The magnitude of the term $\sin \alpha_{\text {eff }}$ still ranges from 0.2 to 0.4 but its maximum is no longer reached at half period but at the transition between the two stages of the dynamic stall 


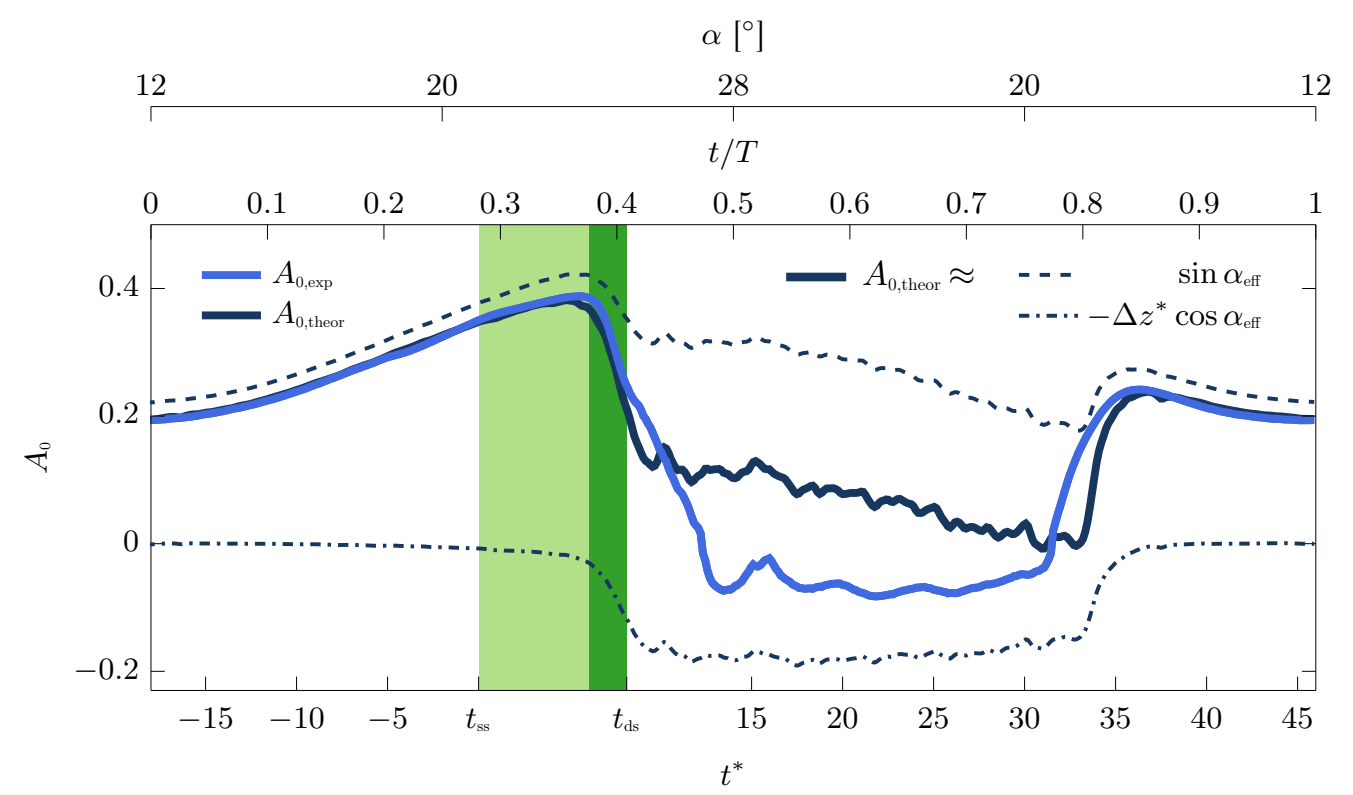

FIG. 11. Comparison of the experimental phase-averaged leading edge suction parameter $\left(A_{0, \mathrm{exp}}\right)$ and the modified theoretical model $\left(A_{0, \text { theor }}\right)$ that takes into account the twofold influence of the shear layer on the effective angle of attack and the effective camber. The influence of different terms in equation (15) are presented by dashed lines.

development. When the flow is attached, the geometric and effective angles of attack are close. When the flow starts to separate and when the shear layer rolls up, the shear layer height increases, reducing the effective angle of attack. This leads to a reduction in the contribution of the first term when the flow progressively separates.

The last term $\Delta z^{\star} \cos \alpha_{\text {eff }}$ in equation (15) is negative throughout the cycle and mirrors the evolution of the average shear layer height presented in figure 4c. The term $\Delta z^{\star} \cos \alpha_{\text {eff }}$ is an order of magnitude lower than the term $\sin \alpha_{\text {eff }}$ (figure 11), and the modified theoretical leading edge suction parameter is dominated by the effective angle of attack.

The new model accurately predicts the experimentally obtained leading edge suction parameter during the attached flow, the primary instability stage, and the vortex formation stage. During the primary instability stage, the model correctly predicts the decreasing slope of the experimental leading edge suction. The modified theory predicts a maximum value at the transition between the primary instability and the vortex formation stage and a decrease of the suction at the leading edge during the latter. Both the value and the timing of the maximum leading edge suction are correctly estimated. When the shear layer starts to roll 
Modelling the interplay between the shear layer and leading edge suction

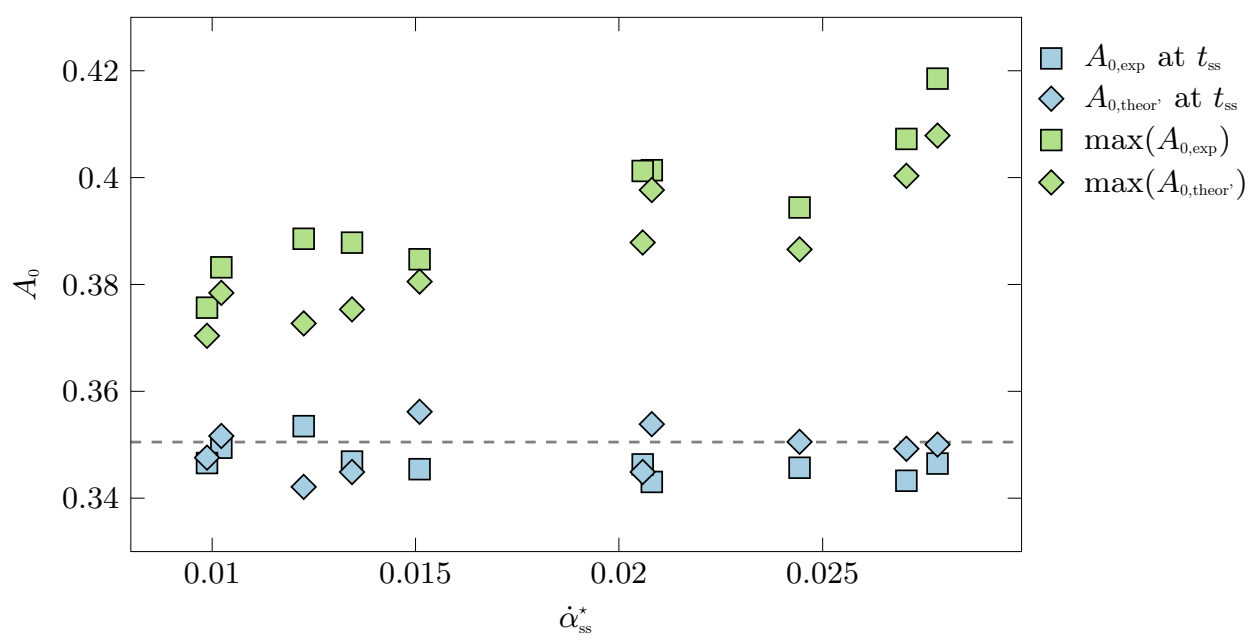

FIG. 12. Values of the leading edge suction parameter, $A_{0}$, at $t_{\mathrm{ss}}$ when the static stall angle is exceeded, and the maximum values during the dynamic stall development for various motion kinematics in function of the normalised effective unsteadiness.

up, the assumptions of the thin airfoil theory reach the boundaries of their validity but the sharp decrease of the leading edge suction parameter is still correctly modelled. During the fully stalled stage, the modified model of the leading edge suction parameter decreases over time but not sufficiently to match the experimental values. At the end of the pitch down motion, during the reattachment stage, the modified theoretical leading edge suction parameter increases and relaxes at the same rate than the experimental leading edge suction parameter, but with a short time lag with respect to the experimental values.

A comparison of the critical values for the experimental and model leading edge suction parameter in function of normalised effective unsteadiness for various motion kinematics is presented in figure 12. The experimental values are presented by squares and the model values taking into account the influence of the shear layer are presented by diamonds. The modified theoretical model predicts well the critical values of the experimental leading edge suction parameter for the range of normalised effective unsteadiness studied.

The general evolution of the modified model of the leading edge suction parameter is dominated by the effective angle of attack. The maximum value of the theoretical leading edge suction parameter is predicted with the correct amplitude at the correct time if the twofold influence of the shear layer is taken into account. The shear layer evolution over the entire airfoil has an influence on the local pressure at the leading edge. The pitch rate has no 
Modelling the interplay between the shear layer and leading edge suction

direct influence on the suction at the leading edge through equation (15) for this range of reduced frequencies $(k \in[0.05: 0.1])$ and at this Reynolds number $\left(R e=9.2 \times 10^{5}\right)$, but it has a strong influence on the flow development on the pitching airfoil and on the shear layer development ${ }^{1,8}$. The influence of the pitch rate on the leading edge suction parameter is thus indirectly taken into account by modelling the effective angle of attack and effective camber variations due to the shear layer development.

\section{CONCLUSIONS}

The dynamic stall life cycle is analysed for a sinusoidal pitching motion by time-resolved velocity field and surface pressure measurements. A two-stage dynamic stall development is independently confirmed by the global velocity field measurements and the local airfoil surface pressure measurements around the leading edge. During the first stage, which is called the primary instability stage, the flow separates near the trailing edge and the separation point moves gradually upstream with increasing angle of attack. During this stage, the leading edge suction increases approximately linearly up to a maximum. During the second stage, the vortex formation stage, the shear layer rolls up to create a dynamic stall vortex that pinches off at stall onset. The leading edge suction decreases rapidly during this stage. The two-stage dynamic stall development is characterised by critical time delays. The time duration of the first stage of the dynamic stall development decreases linearly with increasing motion unsteadiness promoting an earlier roll-up of the shear layer, while the formation of the dynamic stall vortex and the duration of the vortex formation stage are virtually independent of motion unsteadiness. The critical time delays of the two stages are related to the evolution of the shear layer but are independently extracted from the velocity field measurements above the airfoil and the measurement of the leading edge suction parameter derived from the airfoil surface pressure data around the leading edge.

The leading edge suction parameter condenses the spatiotemporal pressure evolution at the leading edge during the dynamic stall life cycle in one robust parameter. The experimentally obtained values of the leading edge suction parameter are analysed for various sinusoidal motions. The maximum leading edge suction increases and is reached sooner for increased normalised effective unsteadiness of the pitching motion. During the vortex formation stage, which starts sooner for higher normalised effective unsteadiness, the gradient of the decrease 
in leading edge suction increases with increasing unsteadiness. The leading edge suction parameter is a practically accessible parameter with high potential to serve as a robust stall onset indicator, but the trigger that leads to the roll-up of the free shear layer is better indicated by motion independent critical values of the chord normal height and the circulation of the shear layer.

An improved model of the leading edge suction parameter is proposed based on thin airfoil theory that links the evolution of the leading edge suction and the shear layer during stall development. The influence of the growth of the shear layer during dynamic stall development on the flow around the airfoil is described in terms of an increase of the effective camber and a decrease of the effective angle of attack of the airfoil. By taking into account this twofold influence of the shear layer development, a new model is derived for the leading edge suction parameter that accurately predicts the value and the timing of the maximum leading edge suction on the pitching airfoil.

\section{ACKNOWLEDGMENTS}

The experimental data was acquired at DLR Göttingen in the Department of Helicopters lead by Markus Raffel. We thank Markus for his support during the time of the measurements and for the opportunity to use the experimental data for further analysis. This work was supported by the Swiss national science foundation under grant number PYAPP2_173652.

\section{REFERENCES}

${ }^{1}$ C. Shih, L. Lourenco, L. Van Dommelen, and A. Krothapalli, "Unsteady flow past an airfoil pitching at a constant rate," AIAA Journal 30, 1153-1161 (1992).

${ }^{2}$ A. Spentzos, G. N. Barakos, K. Badcock, B. Richards, P. Wernert, S. J. Schreck, and M. Raffel, "Investigation of Three-Dimensional Dynamic Stall Using Computational Fluid Dynamics," AIAA Journal 43, 1023-1033 (2005).

${ }^{3}$ W. J. Morris and Z. Rusak, "Stall onset on aerofoils at low to moderately high Reynolds number flows," Journal of Fluid Mechanics 733, 439-472 (2013).

${ }^{4}$ W. J. McCroskey and R. K. Fisher, "Dynamic Stall of Airfoils and Helicopter Rotors," Tech. Rep. (1972). 
${ }^{5}$ L. W. Carr, K. W. McAlister, and W. J. McCroskey, "Analysis of the Development of Dynamic Stall Based on Oscillating Airfoil Experiments," Tech. Rep. (1977).

${ }^{6}$ W. J. McCroskey, "The Phenomenon of Dynamic Stall," Tech. Rep. 81264 (NASA, 1981).

${ }^{7}$ W. J. McCroskey, "Unsteady Airfoils," Annual Review of Fluid Mechanics 14, 285-311 (1982).

${ }^{8}$ L. W. Carr, "Progress in analysis and prediction of dynamic stall," Journal of Aircraft 25, 6-17 (1988).

${ }^{9}$ M. Acharya and H. M. Metwally, "Unsteady pressure field and vorticity production over a pitching airfoil," AIAA Journal 30, 403-411 (1992).

${ }^{10} \mathrm{~T}$. Lee and P. Gerontakos, "Investigation of flow over an oscillating airfoil," Journal of Fluid Mechanics 512, 313-341 (2004).

${ }^{11}$ M. S. H. Boutilier and S. Yarusevych, "Separated shear layer transition over an airfoil at a low Reynolds number," Physics of Fluids 24, 084105 (2012).

${ }^{12}$ R. Gupta and P. J. Ansell, "Unsteady Flow Physics of Airfoil Dynamic Stall," AIAA Journal 0, 1-11 (2018).

${ }^{13}$ A. Lambert and S. Yarusevych, "Effect of angle of attack on vortex dynamics in laminar separation bubbles," Physics of Fluids 31, 064105 (2019).

${ }^{14} \mathrm{~K}$. Mulleners and M. Raffel, "The onset of dynamic stall revisited," Experiments in Fluids 52, 779-793 (2012).

${ }^{15} \mathrm{M}$. Bross and D. Rockwell, "Flow structure on a simultaneously pitching and rotating wing," Journal of Fluid Mechanics 756, 354-383 (2014).

${ }^{16}$ R. Dunne, P. J. Schmid, and B. J. McKeon, "Analysis of Flow Timescales on a Periodically Pitching/Surging Airfoil," AIAA Journal 54, 3421-3433 (2016).

${ }^{17}$ M. Melius, R. B. Cal, and K. Mulleners, "Dynamic stall of an experimental wind turbine blade," Physics of Fluids 28 (2016), 10.1063/1.4942001.

${ }^{18}$ E. C. E. Culler and J. A. N. Farnsworth, "Higher frequencies in stall flutter moment development," Journal of Fluids and Structures 85, 181-198 (2019).

${ }^{19}$ M. R. Visbal and D. J. Garmann, "Analysis of Dynamic Stall on a Pitching Airfoil Using High-Fidelity Large-Eddy Simulations," AIAA Journal 56, 46-63 (2018).

${ }^{20} \mathrm{~S}$. I. Benton and M. R. Visbal, "The onset of dynamic stall at a high, transitional Reynolds number," Journal of Fluid Mechanics 861, 860-885 (2019).

${ }^{21}$ M. E. Rosti, M. Omidyeganeh, and A. Pinelli, "Direct numerical simulation of the flow 
around an aerofoil in ramp-up motion," Physics of Fluids 28, 025106 (2016).

${ }^{22}$ R. Jain, A. LePape, A. Grubb, M. Costes, F. Richez, and M. Smith, "High-resolution computational fluid dynamics predictions for the static and dynamic stall of a finite-span OA209 wing," Journal of Fluids and Structures 78, 126-145 (2018).

${ }^{23}$ A. T. Degani, Q. Li, and J. D. A. Walker, "Unsteady separation from the leading edge of a thin airfoil," Physics of Fluids 8, 704-714 (1996).

${ }^{24}$ B. J. Pruski and R. D. W. Bowersox, "Leading-Edge Flow Structure of a Dynamically Pitching NACA 0012 Airfoil," AIAA Journal 51, 1042-1053 (2013).

${ }^{25} \mathrm{~A}$. Widmann and C. Tropea, "Parameters influencing vortex growth and detachment on unsteady aerodynamic profiles," Journal of Fluid Mechanics 773, 432-459 (2015).

${ }^{26}$ S. I. Benton and M. R. Visbal, "Effects of Leading-Edge Geometry on the Onset of Dynamic Stall," AIAA Journal 56, 4195-4198 (2018).

${ }^{27}$ A. Choudhry, R. Leknys, M. Arjomandi, and R. Kelso, "An insight into the dynamic stall lift characteristics," Experimental Thermal and Fluid Science 58, 188-208 (2014).

${ }^{28}$ H. F. Mller-Vahl, G. Pechlivanoglou, C. N. Nayeri, C. O. Paschereit, and D. Greenblatt, "Matched pitch rate extensions to dynamic stall on rotor blades," Renewable Energy 105, 505-519 (2017).

${ }^{29}$ C. Bose and S. Sarkar, "Investigating chaotic wake dynamics past a flapping airfoil and the role of vortex interactions behind the chaotic transition," Physics of Fluids 30, 047101 (2018).

${ }^{30}$ W. T. Evans and K. W. Mort, "Analysis of computed flow parameters for a set of sudden stalls in low-speed two-dimensional flow," Technical note D-85 (NASA, 1959).

${ }^{31}$ J. G. Leishman and T. S. Beddoes, "A Semi-Empirical Model for Dynamic Stall," Journal Of The American Helicopter Society 34, 3-17 (1989).

${ }^{32}$ L. C. Woods, The Theory of Subsonic Plane Flow (Cambridge university press, 1961).

${ }^{33}$ R. A. M. Niven, A. J .and Galbraith, "Modelling dynamic stall vortex inception at low Mach numbers," The Aeronautical Journal 101, 67-76 (1997).

${ }^{34}$ W. Sheng, R. A. M. Galbraith, and F. N. Coton, "A New Stall-Onset Criterion for Low Speed Dynamic-Stall," Journal of Solar Energy Engineering 128, 461-471 (2005).

${ }^{35}$ K. Ramesh, A. Gopalarathnam, K. Granlund, M. V. Ol, and J. R. Edwards, "Discretevortex method with novel shedding criterion for unsteady aerofoil flows with intermittent leading-edge vortex shedding," Journal of Fluid Mechanics 751, 500-538 (2014). 
${ }^{36}$ S. Narsipur, A. Gopalarathnam, and J. R. Edwards, "Low-Order Model for Prediction of Trailing-Edge Separation in Unsteady Flow," AIAA Journal , 1-17 (2018).

${ }^{37}$ D. Darakananda and J. D. Eldredge, "A versatile taxonomy of low-dimensional vortex models for unsteady aerodynamics," Journal of Fluid Mechanics 858, 917-948 (2019).

${ }^{38}$ K. Ramesh, K. Granlund, M. V. Ol, A. Gopalarathnam, and J. R. Edwards, "Leadingedge flow criticality as a governing factor in leading-edge vortex initiation in unsteady airfoil flows," Theoretical and Computational Fluid Dynamics 32, 109-136 (2018).

${ }^{39}$ J. Katz and A. Plotkin, Low-Speed Aerodynamics, 2nd ed., Cambridge Aerospace Series (Cambridge University Press, 2004).

${ }^{40}$ I. E. Garrick, "Propulsion of a flapping and oscillating airfoil," Tech. Rep. NACA-TR-567 (National Advisory Committee for Aeronautics, Langley Aeronautical Lab.; Langley Field, VA, United States, 1936).

${ }^{41}$ P. G. Wilby, "The aerodynamic characteristics of some new rae blade sections, and their potential influence on rotor performance," in Fifth european rotorcraft and powered lift aircraft forum (Amsterdam, 1979) pp. 11-1-11-16.

${ }^{42}$ J. Deparday and K. Mulleners, "Critical evolution of leading edge suction during dynamic stall," Journal of Physics: Conference Series 1037, 022017 (2018).

${ }^{43}$ K. Mulleners and M. Raffel, "Dynamic stall development," Experiments in Fluids 54, 1469-1477 (2013).

${ }^{44} \mathrm{P}$. Ansell and K. Mulleners, "Multi-scale vortex characteristics of dynamic stall from empirical mode decomposition," AIAA Journal , 1-18 (2019).

${ }^{45}$ D. Garcia, "A fast all-in-one method for automated post-processing of PIV data," Experiments in Fluids 50, 1247-1259 (2010).

${ }^{46}$ L. Graftieaux, M. Michard, and N. Grosjean, "Combining PIV, POD and vortex identification algorithms for the study of unsteady turbulent swirling flows," Measurement Science and Technology 12, 1422-1429 (2001).

${ }^{47}$ C.-M. Ho and P. Huerre, "Perturbed Free Shear Layers," Annual Review of Fluid Mechanics 16, 365-424 (1984).

${ }^{48}$ V. J. Peridier, F. T. Smith, and J. D. A. Walker, "Vortex-induced boundary-layer separation. Part 2. Unsteady interacting boundary-layer theory," Journal of Fluid Mechanics 232, 133-165 (1991).

${ }^{49}$ W. McCroskey, L. Carr, and K. McAlister, "Dynamic Stall Experiments on Oscillating 
Airfoils," AIAA Journal 14, 57-63 (1976).

${ }^{50}$ D. E. Rival, J. Kriegseis, P. Schaub, A. Widmann, and C. Tropea, "Characteristic length scales for vortex detachment on plunging profiles with varying leading-edge geometry," Experiments in Fluids 55, 1660 (2014).

${ }^{51}$ Y. Huang and M. A. Green, "Detection and tracking of vortex phenomena using Lagrangian coherent structures," Experiments in Fluids 56, 147-158 (2015).

${ }^{52}$ M. P. Rockwood, K. Taira, and M. A. Green, "Detecting Vortex Formation and Shedding in Cylinder Wakes Using Lagrangian Coherent Structures," AIAA Journal 55, 15-23 (2016).

${ }^{53}$ S. Krishna, M. A. Green, and K. Mulleners, "Flowfield and Force Evolution for a Symmetric Hovering Flat-Plate Wing," AIAA Journal 56, 1360-1371 (2018).

${ }^{54}$ S. L. Brunton, S. T. M. Dawson, and C. W. Rowley, "State-space model identification and feedback control of unsteady aerodynamic forces," Journal of Fluids and Structures 50, 253-270 (2014).

${ }^{55}$ C. W. Rowley and S. T. Dawson, "Model Reduction for Flow Analysis and Control," Annual Review of Fluid Mechanics 49, 387-417 (2017).

${ }^{56}$ D. Bhattacharjee, M. Hemati, B. Klose, and G. Jacobs, "Optimal Actuator Selection for Airfoil Separation Control," in 2018 Flow Control Conference (American Institute of Aeronautics and Astronautics, Atlanta, Georgia, 2018).

${ }^{57}$ J.-C. Loiseau, B. R. Noack, and S. L. Brunton, "Sparse reduced-order modelling: sensorbased dynamics to full-state estimation," Journal of Fluid Mechanics 844, 459-490 (2018).

${ }^{58} \mathrm{~J}$. Kou and W. Zhang, "Dynamic mode decomposition with exogenous input for datadriven modeling of unsteady flows," Physics of Fluids 31, 057106 (2019).

${ }^{59}$ J. Katz, "A discrete vortex method for the non-steady separated flow over an airfoil," Journal of Fluid Mechanics 102, 315-328 (1981).

${ }^{60}$ A. SureshBabu, K. Ramesh, and A. Gopalarathnam, "Model Reduction in Discrete-Vortex Methods for Unsteady Airfoil Flows," AIAA Journal , 1-14 (2019).

${ }^{61}$ X. Li, L.-H. Feng, and Z.-Y. Li, "Flow mechanism for the effect of pivot point on the aerodynamic characteristics of a pitching airfoil and its manipulation," Physics of fluids 31, 087108 (2019). 